\title{
p62 links the autophagy pathway and the ubiqutin-proteasome system upon ubiquitinated protein degradation
}

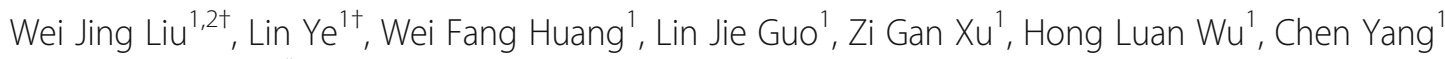
and Hua Feng Liu ${ }^{1 *}$

\footnotetext{
* Correspondence: hf-liu@263.net

${ }^{\dagger}$ Equal contributors

${ }^{1}$ The Institute of Nephrology, Guangdong Medical University, Zhanjiang, Guangdong 524001, China

Full list of author information is available at the end of the article
}

\begin{abstract}
The ubiquitin-proteasome system (UPS) and autophagy are two distinct and interacting proteolytic systems. They play critical roles in cell survival under normal conditions and during stress. An increasing body of evidence indicates that ubiquitinated cargoes are important markers of degradation. p62, a classical receptor of autophagy, is a multifunctional protein located throughout the cell and involved in many signal transduction pathways, including the Keap1-Nrf2 pathway. It is involved in the proteasomal degradation of ubiquitinated proteins. When the cellular p62 level is manipulated, the quantity and location pattern of ubiquitinated proteins change with a considerable impact on cell survival. Altered p62 levels can even lead to some diseases. The proteotoxic stress imposed by proteasome inhibition can activate autophagy through p62 phosphorylation. A deficiency in autophagy may compromise the ubiquitin-proteasome system, since overabundant p62 delays delivery of the proteasomal substrate to the proteasome despite proteasomal catalytic activity being unchanged. In addition, p62 and the proteasome can modulate the activity of HDAC6 deacetylase, thus influencing the autophagic degradation.
\end{abstract}

Keywords: p62, Autophagy, Ubiquitin-proteasome system (UPS), Ubiquitinated protein, Aggresome, Proteostasis, p62 phosphorylation, Keap1-Nrf2 pathway, Histone deacetylase 6 (HDAC6), Mechanistic target of rapamycin complex 1 (mTORC1)

\section{Introduction}

Nearly $30 \%$ of newly synthesized proteins in the cell are misfolded under normal conditions [1]. Two systems that maintain cellular proteostasis are the ubiquitin-proteasome system (UPS) and autophagy. These self-governed systems degrade various substrates, and while they are distinct, a growing body of evidence indicates cooperation between them. They share some ubiquitinated proteins, such as HttQ74, a huntingtin protein in Huntington's disease [2], but also degradation elements, such as p62.

p62 is an autophagy substrate that is used as a reporter of autophagy activity. Recently, p62 was also shown to deliver ubiquitinated proteins, such as tau, to the proteasome for degradation. In addition, it can shuttle between the nucleus and cytoplasm to bind with ubiquitinated cargoes and facilitate nuclear and cytosolic protein quality control. Other 
functions of p62 are gradually being revealed, emphasizing its importance in the proteolytic system. This review focuses on the role of p62 in linking the ubiquitin-proteasome system and autophagy pathway upon ubiquitinated protein degradation (Fig. 1).

\section{Ubiquitin-proteasome system}

The ubiquitin-proteasome system (UPS) plays a critical role in the degradation of short-lived, misfolded and damaged proteins. This is necessary to maintain protein homeostasis, cell cycle control [3], inflammation, oxidative stress, apoptosis [4] and immunity [1]. It even serves a non-proteolytic function in the control of translation [5]. The proteasome is a highly conserved protease complex consisting of two moieties that combine into a diversity of forms: the $20 \mathrm{~S}$ catalytic core particle and the $19 \mathrm{~S}$ or $11 \mathrm{~S}$ regulatory particle(s) (Fig. 2). $20 \mathrm{~S}$ is a barrel-shaped complex that possesses two $\alpha$-rings and two $\beta$-rings, with each ring composed of seven subunits. $\beta 1, \beta 2$ and $\beta 5$ are 3 subunits of each $\beta$-ring, respectively possessing peptidylglutamyl peptide-hydrolyzing or caspase-like activity (PGPH or C-L); trypsin-like activity (T-L); and chymotrypsinlike activity (CT-L) [6]. 19S consists of a lid and a base, which is involved in substrate recognition, deubiquitination, unfolding and further translation into $20 \mathrm{~S}$ for degradation $[7,8]$.

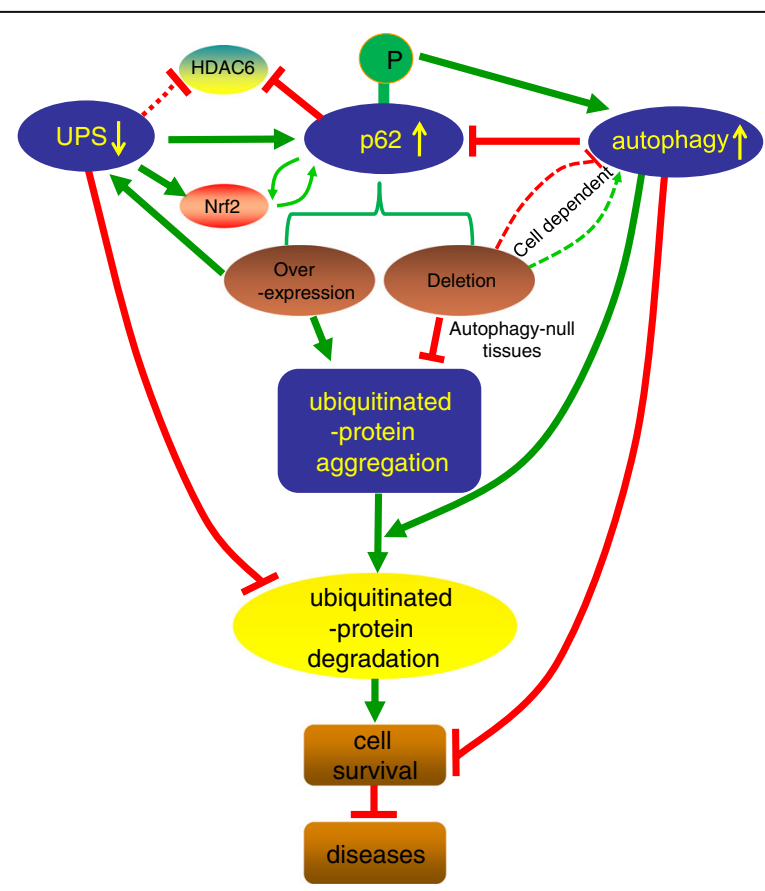

Fig. 1 The interactions of p62 and the UPS, autophagy and ubiquitinated proteins. Upon UPS inhibition, p62 is upregulated and phosphorylated on \$405 and \$409, which can facilitate the degradation of ubiquitinated cargoes via autophagy. p62 synthesis is induced by an increase in Nrf2 following UPS deficiency. The increased p62 competes with Nrf2 for Keap1, and then a p62-Keap1 complex selectively facilitates the ubiquitinated aggregate formation and creates a positive feedback loop with Nrf2. HDAC6 can be activated by the products in UPS (such as K63), but inhibited directly by p62. HDAC6 plays a critical role in ubiquitinated aggregate formation and autophagosome-lysosome fusion, while a ratio of p62 to HDAC6 maintains the homeostasis of autophagic process. Besides inhibiting the degradation of $\mathrm{p} 62$ and ubiquitinated proteins, a deficiency in autophagy also compromises UPS since the increased p62 delays ubiquitinated protein delivery to UPS for degradation. p62 overexpression increases the aggregation of ubiquitinated proteins and has a protective effect on cell survival, while p62 deletion exacerbates cell injury and relates to some diseases by either facilitating or damaging autophagic degradation dependent on the cell type 
A

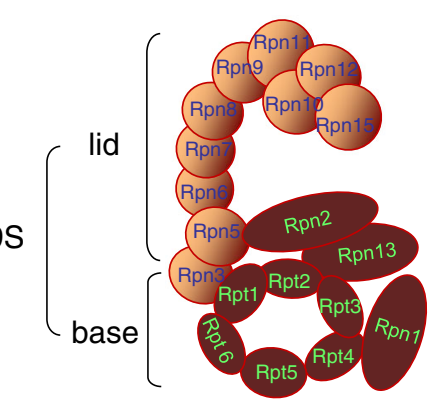

C

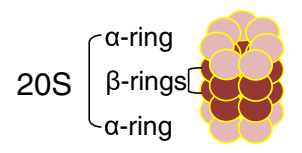

IFN-y

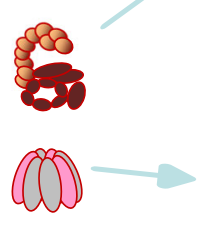

$\beta 11$

$\beta 2 i$

$\beta 5 i$
B<smiles>C[As][As][Mg]</smiles><smiles>c1ccccc1</smiles>

$1 \mathrm{~S}$

top view
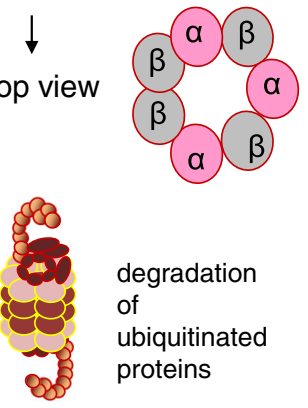

degradation

of

ubiquitinated proteins

$26 S$

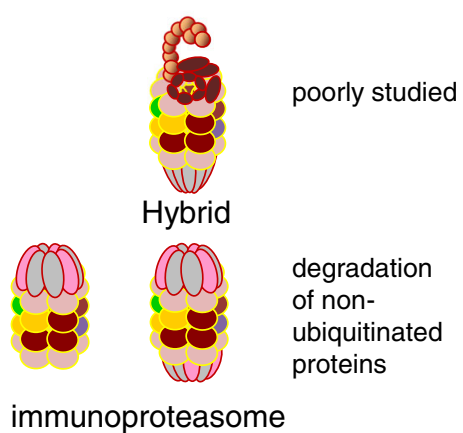

Fig. 2 Structures of the mammalian proteasome. a A simplified model of the proteasome regulatory particle 195. The lid mainly de-ubiquitylates the captured substrates, while the base functions as substrate unfolding and translocation. Rpn11 serves as a de-ubiquitylating enzyme (DUB) en clon cleaving the polyubiquitin chain of substrates. Rpt1-6, an ATPase ring, is involved in substrate protein unfolding and translocation into the channel of the 20S. Rpn13 and Rpn10 (a lid subunit) serve as ubiquitin receptors. Rpn1 can bind to the ubiquitin shuttle receptors and cytoplasmic deubiquitinases. b A simplified model of the proteasome regulatory particle 11S, which is also termed PA28. It is an activator of the proteasome. c Assembly model of mammalian proteasome. $20 \mathrm{~S}$ binding 195 at one or two ends generates the $26 \mathrm{~S}$ proteasome (or 30S), with an ATP-dependent degradation of ubiquitinated substrates. Upon stimulation of interferon- $\gamma($ INF- $\gamma)$, all three active subunits $(\beta 1, \beta 2$ and $\beta 5)$ of the constitutive 205 proteasome are replaced by close-proximity similar subunits ( $\beta 1 \mathrm{i}, \beta 2 \mathrm{i}$ and $\beta 5 \mathrm{i}$, respectively) that bind to $11 \mathrm{~S}$ to generate the immunoproteasome. The immunoproteasome responds to antigen presentation with a non-ATPdependent degradation of non-ubiquitinated proteins

The progress of proteolysis also requires ubiquitin to covalently attach to substrates. This 76-amino acid protein can form an isopeptide bond between its C-terminal glycine (G76) and a lysine residue within the target molecules or ubiquitin itself [9]. Ubiquitination is completed via an enzymatic cascade involving E1 ubiquitin-activating enzyme(s), E2 ubiquitin-conjugating enzyme(s) and E3 ubiquitin ligase(s). Then the ubiquitinated proteins are recognized and degraded by the $26 \mathrm{~S}$ proteasome, which consists of a $20 \mathrm{~S}$ unit with one or two $19 \mathrm{~S}$ units at one or both ends [10].

\section{Autophagy}

Autophagy is a highly evolutionarily conserved degradation system in eukaryotes [11]. It was first considered to be a non-selective bulk system for degrading long-lived proteins and organelles to recycle nutrients and generate energy [12]. Later studies 
showed that autophagy selectively degrades protein aggregates (aggrephagy), peroxisomes (pexophagy), damaged mitochondria (mitophagy), intracellular bacteria and viruses (xenophagy), surplus endoplasmic reticulum (reticulophagy), ribosomes (ribophagy) and mid-body ring structures [13].

The autophagic degradation model of eukaryotes is emerging through more recent research [12]. Autophagy begins with the formation of a phagophore, which is a crescent-shaped double membrane tightly associated with LC3II. The phagophore engulfs adaptor-mediated ubiquitinated substrates to become an autophagosome, which fuses with the lysosome to become an autolysosome with an internal acidic, hydrolytic environment that helps to degrade the content [14]. For content outside the cell, the cell membrane caves to envelop it. This is an endosome, which fuses with an autophagosome to become an amphisome, which in turn fuses with a lysosome to become an autolysosome.

Autophagy-related gene (Atg) proteins play essential roles in autophagy. They are known as the 'core machinery' [15]. More than 40 Atg proteins have been identified as participating in autophagy or autophagy-related processes [16]. p62 and NBR1 (neighbor of BRCA1 gene 1) are two important cargo receptors involved in selective autophagy. They are essential in the formation of ubiquitinated aggregates [17, 18]. NBR1 is twice as large as p62, has a similar domain architecture, and shares several key features with it. Cellular NBR1 is modulated by the autophagic process and does not seem to be influenced by proteasomal degradation [17]. Recent studies have revealed a critical role for autophagy in some human diseases, such as tumors [19], neurodegenerative diseases and aging. Some cell lines, such as podocytes, have high basal autophagy [20].

\section{p62}

p62 was the first selected autophagy adaptor discovered in mammals [11, 21, 22]. It was termed sequestosome 1 (SQSTM 1) by Shin due to its ability to form aggregates [23]. A170 and ZIP are the respective names for the mouse and rat variants.

p62 is a multifunctional protein consisting of an N-terminal Phox-BEM1 domain (PB1), a ZZ-type zinc finger domain, a nuclear localization signal (NLS), an export motif (NES), an LC3-interacting region (LIR), a Keap1-interacting region (KIR), and a C-terminal ubiquitin-associated domain (UBA) [24, 25] (Fig. 3). p62 interacts non-covalently with ubiquitin or polyubiquitin chains via the UBA domain, and then delivers polyubiquitinated cargoes to autophagy via the LIR domain (which is also known as the Atg8 familyinteracting motif), and to the proteasome via the PB1 domain [23, 26]. In addition to a high potential for homo-oligomerization [27, 28], the PB1 domain can also heterooligomerize with NBR1 or other PB1 domain-containing proteins, such as atypical protein kinases Cs ( $\alpha \mathrm{PKCs})$, MEKK3, MEK5, ERK1 and Rpt1, which modulate different signaling pathways and get involved in osteoclastogenesis, angiogenesis and early cardiovascular development or cell polarity [29].

The oligomerization of p62 via the PB1 domain is critical for ubiquitinated protein accumulation in autophagy-null cells [30]. $\alpha \mathrm{PKCs}$ and MEKK3 can activate NF- $\kappa \mathrm{B}$ signaling by binding the PB1 domain of p62, respectively with the assistance of the receptor-interacting protein-1-binding (RIP-binding) ZZ domain and tumor necrosis factor receptor-associated factor 6-binding (TRAF6-binding) TB domain [31, 32]. The p62-TRAF6 complex appears to modulate the ubiquitination of the IKK complex [33]. 


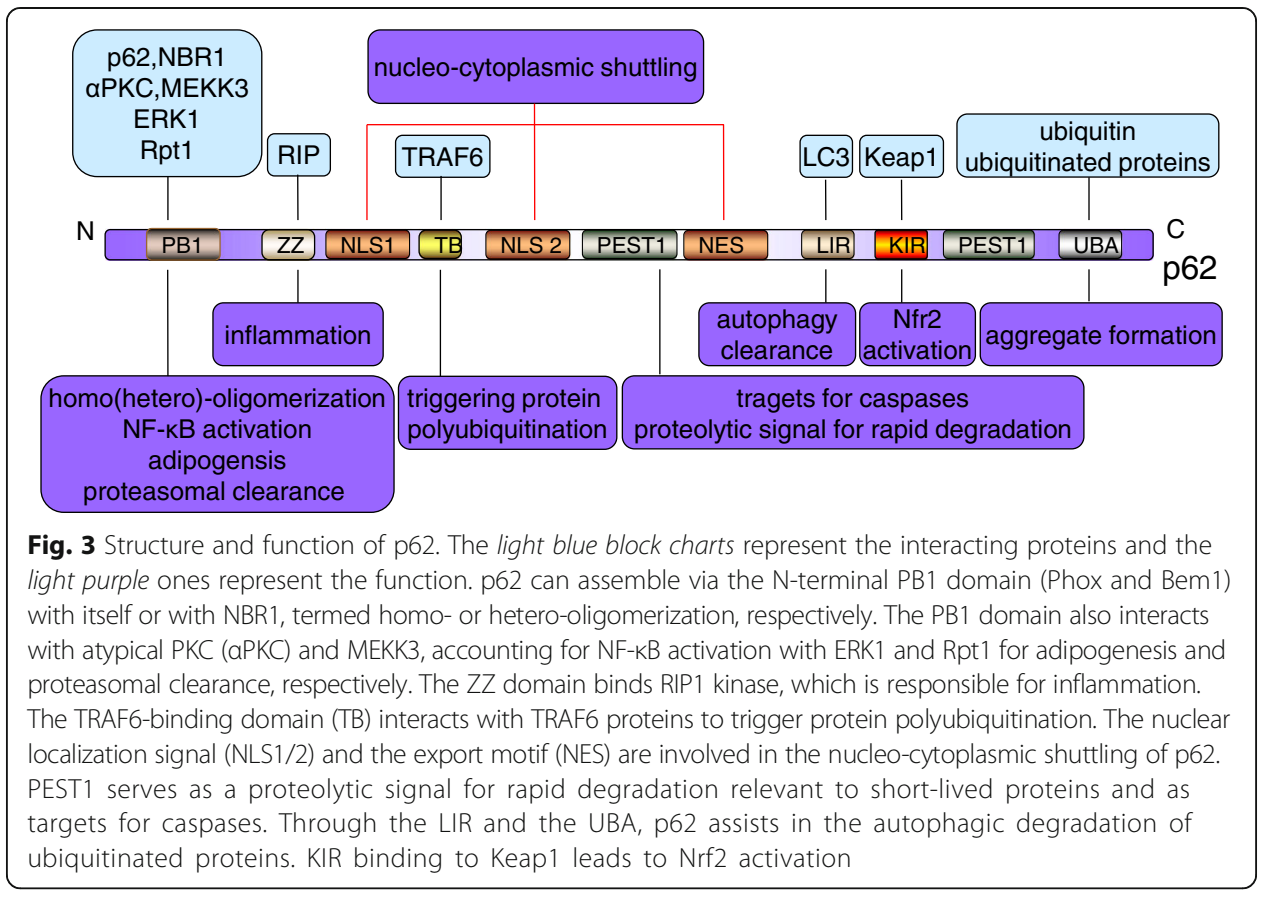

The specific role of MEK5 binding to p62 is actually unclear at present. p62 controls adipogenesis and obesity via interaction with ERK1 [34]. The functions of the other domains will be covered in later chapters.

The intracellular level of p62 is dependent on transcriptional regulation and posttranslational autophagic degradation. The transcription of p62 is modulated by oxidative stress (Nrf2), the Ras/MAPK pathway, the JNK/c-Jun pathway and some chemical compounds (e.g., resveratrol, an inducer of autophagy) [35]. Proteasome inhibition and starvation can also induce p62 synthesis [36]. On the other hand, p62 is modulated by autophagy since it acts as a substrate during autophagic degradation.

\section{Ubiquitinated protein degradation pathway}

Ubiquitination is integral to the proteolytic system. Ubiquitin possesses 76 amino acid residues and is highly conserved from yeast to humans. It also possesses some nonproteolytic functions, including vesicle trafficking via ubiquitination of membrane proteins, protein kinase activation, DNA repair and chromatin dynamics through monoubiquitinated histone. A common mechanism involves ubiquitin or polyubiquitin chains recruiting ubiquitin receptors to perform biological functions [37].

Ubiquitin is involved in three degradation pathways (UPS, lysosome and autophagy). The triage of ubiquitinated proteins is probably based on their location, the ubiquitin chain length and the linkage types. The three degradation pathways may be interdependent on the ubiquitin pool in the cell [38]. Based on the lysine residues inside ubiquitin, seven homogeneous polymer chain linkages can be defined: K6, K11, K27, K29, K33, K48 and K63 [39].

The K48 chain is a classical sign of proteasomal degradation [39]. K63 is a common marker in the autophagy process [40]. However, recent studies revealed that the proteasome seems to accept almost all types of ubiquitin chain, including homogenous, heterogeneous, linear, head-to-tail, single and multi-branched chains and even those with 
mono- or multi-monoubiquitination [39, 41]. It is believed that ubiquitin polymer chains consist of at least four ubiquitin moieties [38]. Autophagy can also accept many types of ubiquitin chain [42].

It should be noted that $\mathrm{K} 48$ is still the common linkage targeting proteins to the proteasome. During proteasomal degradation, the length of the substrate proteins determines whether the process is mediated by mono- or polyubiquitination [43]. The existence of linkage multiplicity implies subtle alterations that contribute to the strength and/or conformation of the interaction between the proteasome and the substrates. Subtle alterations may control their "priority" to the proteasome, resulting in altered degradation rates that give rise to multiple biological functions [39].

The UBA domain of p62 can bind K48 and K63 (with a higher affinity for K63) [40, 44, 45]. UBA phosphorylation may enhance the affinity for K48 and K63 [46]. The longer ubiquitin chains show higher affinity for p62 [23]. K11 and K13 are thought to have non-proteolytic functions [47], and the other polyubiquitin chains have functions that remain unclear.

Determining the overall contribution of UPS and autophagy to ubiquitinated protein degradation is a topic of considerable interest. Studies with subjects ranging from Drosophila to mice suggest that the inactivation of autophagy by pharmacological or genetic inhibition causes the accumulation of ubiquitinated proteins in the brain [48, 49], skeletal muscle, cardiac muscle, pancreatic $\beta$-cells, podocytes and hepatocytes. However, Natura et al. obtained the opposite results [26]. In their study, they compared the turnover dynamics of endogenous ubiquitinated proteins by proteasome and autophagy by assessing the effects of their inhibitors. They found that pharmacological or genetic inhibition of autophagy could not increase the ubiquitinated protein level, although proteasome inhibition by epoximycin did. However, many proteins are degraded by both UPS and autophagy $[2,50]$. Different cell lines may account for this discrepancy.

\section{The role of p62 in autophagy}

\section{The relationship between $\mathrm{p} 62$ and autophagy}

As mentioned previously, p62 delivers ubiquitinated cargoes for autophagic degradation via the C-terminal UBA domain or the LIR domain, and then the PB1 domain promotes the process [26]. Activating autophagy reduces the expression of p62. Pharmacological and genetic inhibition of autophagy can increase the level of p62 in various cell lines (e.g., HeLa cells [22] and neonatal rat ventricular myocytes [51]), tissues (e.g., cardiomyocytes [51], pancreatic $\beta$-cells [52], skeletal muscle [53], liver [54] and central nervous system [48]), and species (e.g., mice [11] and Drosophila [55]).

In HeLa cells, p62 overexpression has no influence on the autophagic vacuole number and p62 knockdown does not have an effect on autophagic flux [2], indicating that p62 is not required for autophagosome formation under basal condition and starvation. However, in some cell lines, overexpressed p62 enhances protein aggregation and has a protective effect on cell survival $[22,56,57]$. Moreover, p62 deletion results in the impaired formation of the LC3-II, aggresome and autophagosome, exacerbating cell injury and lowering cell viability under basal conditions and misfolded protein stress in cardiomyocytes [58]. On the other hand, silencing p62 can also activate autophagy, as evidenced by an increase in the conversion rate of LC3I to LC3II and in the amount of 
multilayered autophagosomes (which may present mis-regulated autophagy) in several carcinoma cell lines. Autophagic cell death is the result [59]. Thus, although the impact of p62 on autophagy is cell-dependent, p62 deletion leads to cell death in almost all of the cell lines.

p62 is localized to ubiquitin-positive inclusions, which is a common phenomenon that can be observed in some diseases, such as neurodegeneration [60]. Collectively, p62 relates to the formation of ubiquitin-positive inclusions and binds LC3II to facilitate autophagic degradation. Some studies demonstrated that a reduced p62 level is accompanied by reduced formation of ubiquitin-positive aggregates in autophagy-null mice, but not in autophagy-normal mice [11]. A similar result was found in Drosophila [55]. The total amount of ubiquitinated protein in p62knockout tissues is less than that found in autophagy-null tissues [11]. Therefore, p62 is critical for the aggregation of ubiquitinated proteins [11, 21, 55]. In addition, there may be other pathways that form ubiquitinated aggregates [2].

\section{p62 as an autophagic flux reporter}

p62 is widely used as a predictor of autophagic flux [2, 61], since it is a thoroughly explored autophagic substrate. However, many factors should be considered when assessing autophagic activity using p62. First, p62 interacts with several signaling molecules, which affects its transcriptional synthesis, increasing the complexity of p62 as an autophagic flux reporter. For example, the KIR domain of p62 binds Keap1 to free Nrf2, which can then induce p62 synthesis [62]. In addition, p62 is a key sensor of the mTOR pathway, in which p62 is induced by amino acid shortage [63]. On the other hand, some agents, such as the phosphatidylinositol 3-kinase (PtdIns3K) inhibitor LY294002, actinomycin D and wortmannin, can inhibit p62 protein synthesis [35, 64].

Another issue is the degradation process. It has been reported that Pan-cathepsin inhibitors and bafilomycin A1 can stop the degradation of p62 [35]. We should also take UPS into account, since the p62 protein level increases upon proteasome inhibition, as described above. Finally, overexpressed p62 (e.g., p62-GFP) tends to selfaggregate, which manifests as an inaccurate reduction in autophagy activity [65]. Therefore, when assessing autophagy flux with p62 analysis, it is advisable to use other assessments as well, such as the mRNA level of p62 and LC3-II turnover.

During starvation, the expression level of p62 does not always inversely correlate with autophagy activity. Not only can autophagy be induced, but p62 transcriptional synthesis is also active upon starvation [63, 66]. p62 is restored to basal levels upon prolonged starvation via transcription upregulation triggered by amino acid shortage, although p62 is reduced by autophagic degradation during the early hours [66].

The significance of $\mathrm{p} 62$ restoration might be the integration of different proteins to fulfill specific functions, since the other domains of p62 get involved in many signal pathways. Besides, p62 knockdown likely activates autophagy through mTORC1 inhibition in response to starvation, since p62 is a positive regulator of mTORC1 [63]. This creates a feed-forward loop in which mTORC1 activation increases p62 levels, further promoting mTORC1 activity. 


\section{A role for $p 62$ in the UPS}

Natura et al. used the proximity ligation assay (PLA) to reveal that p62 and the proteasome are co-localized in situ under basal conditions. They also found that p62 aggregates contain inactive proteasome, ubiquitinated proteins and autophagosome upon proteasome inhibition [26]. It has been shown that p62 can shuttle K63-polyubiquitinated tau for proteasomal degradation [67]. This leads us to explore the relationship between p62 and proteasome.

The N-terminal PB1 domain of p62 might interact with Rpt1 and S5a/Rpn10 of the $26 \mathrm{~S}$ proteasome and collaborate with the C-terminal UBA domain of p62 by binding ubiquitinated proteins to facilitate UPS degradation [26, 66, 68]. p62 continuously undergoes rapid nucleo-cytoplasmic shuttling using its own two nuclear localization signal domains (NLS1 and NLS2) and one nuclear export motif (NES) [63]. p62 is localized in nuclear aggregates [69] and plays a critical role in recruiting the proteasome to the ubiquitinated inclusion in the nucleus. It may also export ubiquitinated cargoes from the nucleus to the cytosol for more efficient degradation [68].

These studies indicate that p62 is also involved in the proteasomal degradation of ubiquitinated proteins in the nucleus via its NLS and NES domains and in the cytosol via its PB1 domain. Therefore, it naturally contributes to both nuclear and cytosolic protein quality control. Besides, the PEST domain serves as a proteolytic signal for rapid degradation, leading to short intracellular half-lives, which may relate to proteasome function [26]. For instance, HS-1-associated protein X-1 (Hax-1) undergoes a fast turnover via the proteasome system through its PEST domain [70].

Pharmacological inhibition of UPS enhances p62 transcription $[26,71]$ and induces the accumulation of ubiquitinated proteins. Inhibiting the proteasome with epoximycin increases the level of p62 far beyond the levels induced by autophagy inhibitors [26]. When p62 is overexpressed, proteasome catalytic activity will be not influenced, although UPS substrates accumulate [2], implying that p62 delays the delivery of ubiquitinated proteins to the UPS for degradation. Moreover, p62 overexpression along with pharmacological inhibition of UPS and/or autophagy does not further increase ubiquitin aggregates. These studies suggest that p62 is not required for all of the ubiquitinated aggregates.

\section{Relationship of p62 with ups and autophagy} Interdependence upon defective proteostasis

Overexpressed p62 can enhance protein aggregation and has a protective effect on cell survival as described above. p62 deletion barely decreases the amount of ubiquitinated puncta in autophagy normal cells. Although p62 is not necessary for all the formation of ubiquitinated aggregation, it still plays a crucial role in aggregate degradation.

Proteasome inhibition can activate autophagy, in which p62 is the bridge [72]. First, proteotoxic stress imposed by proteasome inhibition can induce p62 phosphorylation at serine 405 (S405 in the UBA domain, which is equivalent to S403 in human) and S409 through ULK1/Atg1, which modulates its binding to ubiquitinated proteins [73]. This increased affinity can stabilize ubiquitinated proteins in the sequestosome, which, in turn, prevents p62 dephosphorylation and leads to efficient degradation of the protein aggregates [74]. S409 phosphorylation is essential for the autophagic degradation 
of ubiquitinated proteins, recruitment of autophagy machinery proteins and facilitation of S405 phosphorylation by ULK1, casein kinase 2 (CK2) [29] or TBK-1 [46]. Furthermore, proteasome deficiency upregulates p62 transcription [36]. Finally, proteasome deficiency can induce adaptive transcriptional activation of Nrf2, which can induce p62 synthesis [35].

The S351 of KIR is phosphorylated, leading to a rising affinity of p62 for Keap1 and followed by sequestration of Keap1 on the cargoes [75]. Subsequently, Nrf2 is stabilized and shuttled into the nucleus to function. The p62-Keap1 complex selectively facilitates aggregate formation entrapped by autophagosomes [76] and creates a positive feedback loop with Nrf2 [62, 77].

NF-E2-related factor 2 (Nrf2), a transcription factor that controls the expression of an abundance of anti-oxidant genes, is degraded by the proteasome via the Cul3Keap1-E3 ligase complex under basal conditions. However, during oxidative stress, p62 expression is upregulated by the nuclear import of Nrf2 resulting from the blocked interaction between Keap1 (a negative regulator of Nrf2) and Nrf2. The increased p62 can compete with Nrf2 for Keap1 at the Nrf2-binding site, forming a positive feedback loop [62, 78]. All of the investigations suggest that cellular defense mechanisms are networked to fight against defective proteostasis and p62 is a center regulator.

While proteasome deficiency enhances autophagy, autophagy inactivation compromises the ubiquitin-proteasome system due to surplus $\mathrm{p} 62$, which delays proteasomal substrate delivery to the proteasome with no changes shown in proteasomal catalytic activity [2]. There is another opinion that pharmacological or genetic inhibition of autophagy can activate the proteasome, as evidenced by an increase in proteasomal activities and the upregulation of proteasomal subunits under nutrient-deficient conditions [79]. It is plausible that the situation occurring in the cell upon starvation is much different from other conditions, such as in the state of overabundant proteins. For instance, p62 can no longer reflect autophagic activity during starvation as mentioned above. Phosphorylation of p62 by ULK1 may enhance autophagic clearance, but that does not occur during starvation, despite its role in canonical autophagy signaling [73].

\section{Cooperation in aggresome degradation}

The terms 'aggresome,' aggregate', and 'inclusion bodies' are used to describe misfolded protein granules in cells. In 1998, the aggresome was defined as a 'pericentriolar membrane-free, cytoplasmic inclusion containing misfolded ubiquitinated proteins encased in a cage of intermediate filament proteins that co-localize with the microtubule organizing center (MTOC), in which the autophagosome and lysosome fuse [80]. The 'aggregate protein' can be concentrated to become the aggresome from the periphery to the peri-nucleus. The 'inclusion bodies' have a broader definition that is not microtubule dependent [81]. This terminology is challenged because it gives the impression of a static state, while many of the bodies are actually highly dynamic and reversible. The term 'dynamic droplets' was suggested to describe the dynamic liquidphase structures as opposed to the solid-phase structures (amyloid-like aggregates) [82]. Further investigation is required to make a final affirmative determination.

Although the aggresome was initially used to describe the disease-associated inclusion bodies formed in neurodegenerative diseases, e.g., Lewy bodies in Parkinson's disease and hyaline inclusion bodies in amyotrophic lateral sclerosis (ALS), the 
relevance of the aggresome to inclusions in disease is still disputable [77]. Almost a decade ago, disease-related proteins, such as huntingtin (Htt), were found to form a different pattern compared with the misfolded protein upon proteasome inhibition [83], i.e., periphery versus peri-nuclear, despite other similar biological characteristics.

There is a common consensus that misfolded proteins aggregate and are concentrated in the aggresome, which is removed via the autophagy-lysosome pathway [77]. Degradation is strongly based on the activity of histone deacetylase 6 (HDAC6), which also plays a pivotal role in aggresome formation [84]. Acetylated cortactin becomes cortactin via HDCA6 deacetylase activity, and the latter interacts with F-actin to form cortactin-F-actin assemblies that are recruited to the MTOC, promoting autophagosome and lysosome fusion and substrate clearance. p62 can modulate this process by directly inhibiting HDAC6 activity and facilitating removal of the cortactin-F-actin assembly to MTOC, which seems paradoxical [84]. Some observations suggest that loss of p62 leads to cortactin-F-actin assemblies remaining localized in the periphery and ubiquitinated protein accumulation [85]. HDAC6 knockdown leads to failure of fusion between the autophagosome and lysosome and subsequent protein aggregation [86]. p62 can facilitate protein aggregation and also modulate protein transport to the processing site, while HDAC6 facilitates autophagosome-lysosome fusion. The ratio of p62 to HDAC6 maintains the homeostasis of the autophagic process. The proteasome can also modulate aggresome degradation: Poh1, a subunit of 19S, cleaves ubiquitin chains from the substrates, and subsequently the products and K63 activate HDAC6 [87]. While proteasome inhibition imposes proteotoxic stress, the cell fate (survival or death) in response to an altered ratio of p62 to HDAC6 remains unclear.

\section{Conclusion}

Because the UPS, autophagy and p62 are the interdependent elements of the protein quality control system, they must act in a networked manner to maintain proteostasis. p62 may serve as an integration center for multiple functions, including the formation of the autophagosome, the delivery of ubiquitinated proteins to the proteasome, and aggregate formation for autophagic clearance. It is also involved in several signaling pathways [88, 89]. In addition, it has been shown that p62 can inhibit ATP- and ubiquitin-independent LC3 degradation by the proteasome [90]. p62 is also involved in many diseases. For instance, the mutation of the UBA domain in p62 leads to Paget's disease [91]. p62 and autophagy synergize to promote tumor growth [92], and p62 selectively binds mutant SOD1 to form aggregates in model systems of familial amyotrophic lateral sclerosis $[25,57]$. Therefore, p62 could be a promising strategic target for treatment of certain pathological conditions.

\footnotetext{
Abbreviations

HDAC6: Histone deacetylase 6; MTOC: The microtubule organizing center; mTORC1: Mechanistic target of rapamycin complex 1; Nrf2: NF-E2-related factor 2; UPS: The ubiquitin-proteasome system; aPKCs: Atypical protein kinases Cs

Acknowledgements

Not applicable.

Funding

This study was supported by the National Natural Science Foundation of China (No. 81570656 and 81470959), Natural Science Foundation of Guangdong Province (No. 2014A030313540), Medical Scientific Research Foundation of Guangdong Province (No. A2015138 and A2014480) and the Administration of Traditional Chinese Medicine of Guangdong Province (No. 20141153).
} 
Availability of data and materials

Not applicable.

\section{Authors' contributions}

WJL and LY conceived the structure and wrote this manuscript; WFH, LG and ZGX designed the figures; HLW wrote about the ubiquitinated protein degradation pathways. CY and HFL improved the structure and language of the manuscript; All authors read and approved the final manuscript.

\section{Competing interests}

The authors declare that they have no competing interests.

\section{Consent for publication}

Not applicable.

\section{Ethics approval and consent to participate}

Not applicable.

\section{Author details}

The Institute of Nephrology, Guangdong Medical University, Zhanjiang, Guangdong 524001, China. ${ }^{2}$ Key Laboratory of Chinese Internal Medicine of Ministry of Education and Beijing, Dongzhimen Hospital Affiliated to Beijing University of Chinese Medicine, Beijing 100700, China.

Received: 4 December 2016 Accepted: 7 December 2016

Published online: 13 December 2016

\section{References}

1. Schubert U, Anton LC, Gibbs J, Norbury CC, Yewdell JW, Bennink JR. Rapid degradation of a large fraction of newly synthesized proteins by proteasomes. Nature. 2000;404:770-4.

2. Korolchuk VI, Mansilla A, Menzies FM, Rubinsztein DC. Autophagy inhibition compromises degradation of ubiquitin-proteasome pathway substrates. Mol Cell. 2009;33:517-27.

3. Castro A, Bernis C, Vigneron S, Labbe JC, Lorca T. The anaphase-promoting complex: a key factor in the regulation of cell cycle. Oncogene. 2005;24:314-25.

4. Orlowski RZ. The role of the ubiquitin-proteasome pathway in apoptosis. Cell Death Differ. 1999;6:303-13.

5. Yao T, Ndoja A. Regulation of gene expression by the ubiquitin-proteasome system. Semin Cell Dev Biol. 2012;23:523-9.

6. Goldberg AL. Functions of the proteasome: from protein degradation and immune surveillance to cancer therapy. Biochem Soc Trans. 2007:35:12-7.

7. Verma R, Aravind L, Oania R, McDonald WH, Yates 3rd JR, Koonin EV, Deshaies RJ. Role of Rpn11 metalloprotease in deubiquitination and degradation by the 265 proteasome. Science. 2002;298:611-5.

8. Hanna J, Finley D. A proteasome for all occasions. FEBS Lett. 2007;581:2854-61.

9. Hershko A, Ciechanover A. The ubiquitin system. Annu Rev Biochem. 1998;67:425-79.

10. Hochstrasser M. Lingering mysteries of ubiquitin-chain assembly. Cell. 2006;124:2734

11. Komatsu M, Waguri S, Koike M, Sou YS, Ueno T, Hara T, Mizushima N, Iwata J, Ezaki J, Murata S, Hamazaki J, Nishito Y, lemura S, Natsume T, Yanagawa T, Uwayama J, Warabi E, Yoshida H, Ishii T, Kobayashi A, Yamamoto M, Yue Z, Uchiyama Y, Kominami E, Tanaka K. Homeostatic levels of p62 control cytoplasmic inclusion body formation in autophagy-deficient mice. Cell. 2007;131:1149-63.

12. Yang Z, Klionsky DJ. Eaten alive: a history of macroautophagy. Nat Cell Biol. 2010;12:814-22.

13. Rogov $V$, Dotsch $V$, Johansen $T$, Kirkin $V$. Interactions between autophagy receptors and ubiquitin-like proteins form the molecular basis for selective autophagy. Mol Cell. 2014;53:167-78.

14. Wilkinson DS, Jariwala JS, Anderson E, Mitra K, Meisenhelder J, Chang JT, Ideker T, Hunter T, Nizet V, Dillin A, Hansen M. Phosphorylation of LC3 by the Hippo kinases STK3/STK4 is essential for autophagy. Mol Cell. 2015;57:55-68.

15. Xie Z, Klionsky DJ. Autophagosome formation: core machinery and adaptations. Nat Cell Biol. 2007;9:1102-9.

16. Klionsky DJ, Schulman BA. Dynamic regulation of macroautophagy by distinctive ubiquitin-like proteins. Nat Struct Mol Biol. 2014;21:336-45.

17. Lamark T, Kirkin V, Dikic I, Johansen T. NBR1 and p62 as cargo receptors for selective autophagy of ubiquitinated targets. Cell Cycle. 2009;8:1986-90.

18. Komatsu M, Ichimura Y. Physiological significance of selective degradation of p62 by autophagy. FEBS Lett. 2010;584:1374-8

19. Wei H, Guan JL. Blocking tumor growth by targeting autophagy and SQSTM1 in vivo. Autophagy. 2015:11:854-5.

20. Liu WJ, Luo MN, Tan J, Chen W, Huang LZ, Yang C, Pan Q, Li B, Liu HF. Autophagy activation reduces renal tubular injury induced by urinary proteins. Autophagy. 2014;10:243-56.

21. Pankiv S, Clausen TH, Lamark T, Brech A, Bruun JA, Outzen H, Overvatn A, Bjorkoy G, Johansen T. p62/SQSTM1 binds directly to Atg8/LC3 to facilitate degradation of ubiquitinated protein aggregates by autophagy. J Biol Chem. 2007:282:24131-45.

22. Bjorkoy G, Lamark T, Brech A, Outzen H, Perander M, Overvatn A, Stenmark H, Johansen T. p62/SQSTM1 forms protein aggregates degraded by autophagy and has a protective effect on huntingtin-induced cell death. J Cell Biol. 2005;171:603-14.

23. Shin J. P62 and the sequestosome, a novel mechanism for protein metabolism. Arch Pharm Res. 1998;21:629-33.

24. Wang X, Terpstra EJ. Ubiquitin receptors and protein quality control. J Mol Cell Cardiol. 2013;55:73-84.

25. Lin X, Li S, Zhao Y, Ma X, Zhang K, He X, Wang Z. Interaction domains of p62: a bridge between p62 and selective autophagy. DNA Cell Biol. 2013;32:220-7. 
26. Myeku N, Figueiredo-Pereira ME. Dynamics of the degradation of ubiquitinated proteins by proteasomes and autophagy: association with sequestosome 1/p62. J Biol Chem. 2011;286:22426-40.

27. Kirkin V, McEwan DG, Novak I, Dikic I. A role for ubiquitin in selective autophagy. Mol Cell. 2009;34:259-69.

28. Johansen T, Lamark T. Selective autophagy mediated by autophagic adapter proteins. Autophagy. 2011;7:279-96.

29. Moscat J, Diaz-Meco MT, Albert A, Campuzano S. Cell signaling and function organized by PB1 domain interactions. Mol Cell. 2006;23:631-40

30. Ichimura Y, Kumanomidou T, Sou YS, Mizushima T, Ezaki J, Ueno T, Kominami E, Yamane T, Tanaka K, Komatsu M. Structural basis for sorting mechanism of p62 in selective autophagy. J Biol Chem. 2008;283:22847-57.

31. Sanz L, Sanchez P, Lallena MJ, Diaz-Meco MT, Moscat J. The interaction of p62 with RIP links the atypical PKCs to NF-kappaB activation. EMBO J. 1999;18:3044-53.

32. Nakamura K, Kimple AJ, Siderovski DP, Johnson GL. PB1 domain interaction of p62/sequestosome 1 and MEKK3 regulates NF-kappaB activation. J Biol Chem. 2010;285:2077-89.

33. Wooten MW, Geetha T, Seibenhener ML, Babu JR, Diaz-Meco MT, Moscat J. The p62 scaffold regulates nerve growth factor-induced NF-kappaB activation by influencing TRAF6 polyubiquitination. J Biol Chem. 2005;280:35625-9.

34. Lee SJ, Pfluger PT, Kim JY, Nogueiras R, Duran A, Pages G, Pouyssegur J, Tschop MH, Diaz-Meco MT, Moscat J. A functional role for the p62-ERK1 axis in the control of energy homeostasis and adipogenesis. EMBO Rep. 2010;11:226-32.

35. Puissant A, Fenouille N, Auberger P. When autophagy meets cancer through p62/SQSTM1. Am J Cancer Res. 2012:2:397-413.

36. Thompson HG, Harris JW, Wold BJ, Lin F, Brody JP. p62 overexpression in breast tumors and regulation by prostate-derived Ets factor in breast cancer cells. Oncogene. 2003;22:2322-33.

37. Chen ZJ, Sun $\sqcup$. Nonproteolytic functions of ubiquitin in cell signaling. Mol Cell. 2009;33:275-86.

38. Claque MJ, Urbe S. Ubiquitin: same molecule, different degradation pathways. Cell. 2010;143:682-5.

39. Ciechanover A, Stanhill A. The complexity of recognition of ubiquitinated substrates by the 265 proteasome. Biochim Biophys Acta. 1843:2014:86-96.

40. Tan JM, Wong ES, Kirkpatrick DS, Pletnikova O, Ko HS, Tay SP, Ho MW, Troncoso J, Gygi SP, Lee MK, Dawson VL, Dawson TM, Lim KL. Lysine 63-linked ubiquitination promotes the formation and autophagic clearance of protein inclusions associated with neurodegenerative diseases. Hum Mol Genet. 2008;17:431-9.

41. Kravtsova-Ivantsiv Y, Sommer T, Ciechanover A. The lysine48-based polyubiquitin chain proteasomal signal: not a single child anymore. Angew Chem Int Ed Engl. 2013;52:192-8.

42. Schreiber A, Peter M. Substrate recognition in selective autophagy and the ubiquitin-proteasome system. Biochim Biophys Acta. 1843;2014:163-81.

43. Shabek N, Herman-Bachinsky Y, Buchsbaum S, Lewinson O, Haj-Yahya M, Hejjaoui M, Lashuel HA, Sommer T, Brik A, Ciechanover A. The size of the proteasomal substrate determines whether its degradation will be mediated by mono- or polyubiquitylation. Mol Cell. 2012;48:87-97.

44. Long J, Gallagher TR, Cavey JR, Sheppard PW, Ralston SH, Layfield R, Searle MS. Ubiquitin recognition by the ubiquitin-associated domain of p62 involves a novel conformational switch. J Biol Chem. 2008;283:5427-40.

45. Wooten MW, Geetha T, Babu JR, Seibenhener ML, Peng J, Cox N, Diaz-Meco MT, Moscat J. Essential role of sequestosome 1/p62 in regulating accumulation of Lys63-ubiquitinated proteins. J Biol Chem. 2008:283:6783-9.

46. Pilli M, Arko-Mensah J, Ponpuak M, Roberts E, Master S, Mandell MA, Dupont N, Ornatowski W, Jiang S, Bradfute SB, Bruun JA, Hansen TE, Johansen T, Deretic V. TBK-1 promotes autophagy-mediated antimicrobial defense by controlling autophagosome maturation. Immunity. 2012;37:223-34.

47. Behrends C, Harper JW. Constructing and decoding unconventional ubiquitin chains. Nat Struct Mol Biol. 2011:18:520-8.

48. Hara T, Nakamura K, Matsui M, Yamamoto A, Nakahara Y, Suzuki-Migishima R, Yokoyama M, Mishima K, Saito I, Okano H, Mizushima N. Suppression of basal autophagy in neural cells causes neurodegenerative disease in mice. Nature. 2006;441:885-9.

49. Komatsu M, Waguri S, Chiba T, Murata S, Iwata J, Tanida I, Ueno T, Koike M, Uchiyama Y, Kominami E, Tanaka K. Loss of autophagy in the central nervous system causes neurodegeneration in mice. Nature. 2006;441:880-4.

50. Webb JL, Ravikumar B, Atkins J, Skepper JN, Rubinsztein DC. Alpha-Synuclein is degraded by both autophagy and the proteasome. J Biol Chem. 2003;278:25009-13.

51. Tian Z, Wang C, Hu C, Tian Y, Liu J, Wang X. Autophagic-lysosomal inhibition compromises ubiquitin-proteasome system performance in a p62 dependent manner in cardiomyocytes. PLoS One. 2014;9:e100715.

52. Ebato C, Uchida T, Arakawa M, Komatsu M, Ueno T, Komiya K, Azuma K, Hirose T, Tanaka K, Kominami E, Kawamori R, Fujitani Y, Watada H. Autophagy is important in islet homeostasis and compensatory increase of beta cell mass in response to high-fat diet. Cell Metab. 2008;8:325-32.

53. Masiero E, Agatea L, Mammucari C, Blaauw B, Loro E, Komatsu M, Metzger D, Reggiani C, Schiaffino S, Sandri M. Autophagy is required to maintain muscle mass. Cell Metab. 2009;10:507-15.

54. Komatsu M, Waguri S, Ueno T, Iwata J, Murata S, Tanida I, Ezaki J, Mizushima N, Ohsumi Y, Uchiyama Y, Kominami E, Tanaka K, Chiba T. Impairment of starvation-induced and constitutive autophagy in Atg7-deficient mice. J Cell Biol. 2005;169:425-34.

55. Nezis IP, Simonsen A, Sagona AP, Finley K, Gaumer S, Contamine D, Rusten TE, Stenmark H, Brech A. Ref(2)P, the Drosophila melanogaster homologue of mammalian p62, is required for the formation of protein aggregates in adult brain. J Cell Biol. 2008;180:1065-71.

56. Gal J, Strom AL, Kilty R, Zhang F, Zhu H. p62 accumulates and enhances aggregate formation in model systems of familial amyotrophic lateral sclerosis. J Biol Chem. 2007;282:11068-77.

57. Paine MG, Babu JR, Seibenhener ML, Wooten MW. Evidence for p62 aggregate formation: role in cell survival. FEBS Lett. 2005;579:5029-34. 
58. Su H, Wang X. Autophagy and p62 in cardiac protein quality control. Autophagy. 2011;7:1382-3.

59. Nihira K, Miki Y, Ono K, Suzuki T, Sasano H. An inhibition of p62/SQSTM1 caused autophagic cell death of several human carcinoma cells. Cancer Sci. 2014;105:568-75.

60. Knaevelsrud H, Simonsen A. Fighting disease by selective autophagy of aggregate-prone proteins. FEBS Lett. 2010;584:2635-45.

61. Mizushima N, Yoshimori T, Levine B. Methods in mammalian autophagy research. Cell. 2010;140:313-26.

62. Jain A, Lamark T, Sjottem E, Larsen KB, Awuh JA, Overvatn A, McMahon M, Hayes JD, Johansen T. p62/SQSTM1 is a target gene for transcription factor NRF2 and creates a positive feedback loop by inducing antioxidant response element-driven gene transcription. J Biol Chem. 2010;285:22576-91.

63. Duran A, Amanchy R, Linares JF, Joshi J, Abu-Baker S, Porollo A, Hansen M, Moscat J, Diaz-Meco MT. p62 is a key regulator of nutrient sensing in the mTORC1 pathway. Mol Cell. 2011;44:134-46.

64. Seglen PO, Gordon PB. 3-Methyladenine: specific inhibitor of autophagic/lysosomal protein degradation in isolated rat hepatocytes. Proc Natl Acad Sci U S A. 1982;79:1889-92.

65. Pircs K, Nagy P, Varga A, Venkei Z, Erdi B, Hegedus K, Juhasz G. Advantages and limitations of different p62-based assays for estimating autophagic activity in Drosophila. PLoS One. 2012;7:e44214.

66. Sahani MH, Itakura E, Mizushima N. Expression of the autophagy substrate SQSTM1/p62 is restored during prolonged starvation depending on transcriptional upregulation and autophagy-derived amino acids. Autophagy. 2014;10:431-41.

67. Babu JR, Geetha T, Wooten MW. Sequestosome 1/p62 shuttles polyubiquitinated tau for proteasomal degradation. J Neurochem. 2005;94:192-203.

68. Pankiv S, Lamark T, Bruun JA, Overvatn A, Bjorkoy G, Johansen T. Nucleocytoplasmic shuttling of p62/SQSTM1 and its role in recruitment of nuclear polyubiquitinated proteins to promyelocytic leukemia bodies. J Biol Chem. 2010; 285:5941-53.

69. Pikkarainen M, Hartikainen $\mathrm{P}$, Soininen $H$, Alafuzoff I. Distribution and pattern of pathology in subjects with familial or sporadic late-onset cerebellar ataxia as assessed by p62/sequestosome immunohistochemistry. Cerebellum. 2011;10:720-31.

70. Li B, Hu Q, Xu R, Ren H, Fei E, Chen D, Wang G. Hax-1 is rapidly degraded by the proteasome dependent on its PEST sequence. BMC Cell Biol. 2012;13:20.

71. Choe JY, Jung HY, Park KY, Kim SK. Enhanced p62 expression through impaired proteasomal degradation is involved in caspase-1 activation in monosodium urate crystal-induced interleukin-1b expression. Rheumatology (Oxford). 2014;53:1043-53.

72. Cortes CJ, La Spada AR. Autophagy in polyglutamine disease: imposing order on disorder or contributing to the chaos? Mol Cell Neurosci. 2015;66:53-61.

73. Lim J, Lachenmayer ML, Wu S, Liu W, Kundu M, Wang R, Komatsu M, Oh YJ, Zhao Y, Yue Z. Proteotoxic stress induces phosphorylation of p62/SQSTM1 by ULK1 to regulate selective autophagic clearance of protein aggregates. PLoS Genet. 2015;11:e1004987.

74. Matsumoto G, Wada K, Okuno M, Kurosawa M, Nukina N. Serine 403 phosphorylation of p62/SQSTM1 regulates selective autophagic clearance of ubiquitinated proteins. Mol Cell. 2011;44:279-89.

75. Ichimura Y, Waguri S, Sou YS, Kageyama S, Hasegawa J, Ishimura R, Saito T, Yang Y, Kouno T, Fukutomi T, Hoshii T, Hirao A, Takagi K, Mizushima T, Motohashi H, Lee MS, Yoshimori T, Tanaka K, Yamamoto M, Komatsu M. Phosphorylation of p62 activates the Keap1-Nrf2 pathway during selective autophagy. Mol Cell. 2013;51:618-31.

76. Fan W, Tang Z, Chen D, Moughon D, Ding X, Chen S, Zhu M, Zhong Q. Keap1 facilitates p62-mediated ubiquitin aggregate clearance via autophagy. Autophagy. 2010;6:614-21.

77. Kageyama S, Sou YS, Uemura T, Kametaka S, Saito T, Ishimura R, Kouno T, Bedford L, Mayer RJ, Lee MS, Yamamoto M, Waguri S, Tanaka K, Komatsu M. Proteasome dysfunction activates autophagy and the Keap1-Nrf2 pathway. J Biol Chem. 2014;289:24944-55.

78. Su H, Wang X. p62 Stages an interplay between the ubiquitin-proteasome system and autophagy in the heart of defense against proteotoxic stress. Trends Cardiovasc Med. 2011;21:224-8.

79. Wang XJ, Yu J, Wong SH, Cheng AS, Chan FK, Ng SS, Cho CH, Sung JJ, Wu WK. A novel crosstalk between two major protein degradation systems: regulation of proteasomal activity by autophagy. Autophagy. 2013;9:1500-8.

80. Johnston JA, Ward CL, Kopito RR. Aggresomes: a cellular response to misfolded proteins. J Cell Biol. 1998;143:1883-98.

81. Kopito RR. Aggresomes, inclusion bodies and protein aggregation. Trends Cell Biol. 2000;10:524-30.

82. Amen T, Kaganovich D. Dynamic droplets: the role of cytoplasmic inclusions in stress, function, and disease. Cell Mol Life Sci. 2015;72:401-15.

83. Kaganovich D, Kopito R, Frydman J. Misfolded proteins partition between two distinct quality control compartments. Nature. 2008;454:1088-95.

84. Yan J, Seibenhener ML, Calderilla-Barbosa L, Diaz-Meco MT, Moscat J, Jiang J, Wooten MW, Wooten MC. SQSTM1/ p62 interacts with HDAC6 and regulates deacetylase activity. PLoS One. 2013;8:e76016.

85. Seibenhener ML, Babu JR, Geetha T, Wong HC, Krishna NR, Wooten MW. Sequestosome 1/p62 is a polyubiquitin chain binding protein involved in ubiquitin proteasome degradation. Mol Cell Biol. 2004;24:8055-68.

86. Lee JY, Koga H, Kawaguchi Y, Tang W, Wong E, Gao YS, Pandey UB, Kaushik S, Tresse E, Lu J, Taylor JP, Cuervo AM, Yao TP. HDAC6 controls autophagosome maturation essential for ubiquitin-selective quality-control autophagy. EMBO J. 2010;29:969-80.

87. Hao R, Nanduri P, Rao Y, Panichelli RS, Ito A, Yoshida M, Yao TP. Proteasomes activate aggresome disassembly and clearance by producing unanchored ubiquitin chains. Mol Cell. 2013;51:819-28.

88. Hayashi K, Dan K, Goto F, Tshuchihashi N, Nomura Y, Fujioka M, Kanzaki S, Ogawa K. The autophagy pathway maintained signaling crosstalk with the Keap1-Nrf2 system through p62 in auditory cells under oxidative stress. Cell Signal. 2015;27:382-93. 
89. Chang KH, Sengupta A, Nayak RC, Duran A, Lee SJ, Pratt RG, Wellendorf AM, Hill SE, Watkins M, Gonzalez-Nieto D, Aronow BJ, Starczynowski DT, Civitelli R, Diaz-Meco MT, Moscat J, Cancelas JA. p62 is required for stem cell/ progenitor retention through inhibition of IKK/NF-kappaB/CcI4 signaling at the bone marrow macrophageosteoblast niche. Cell Rep. 2014;9:2084-97.

90. Gao Z, Gammoh N, Wong PM, Erdjument-Bromage H, Tempst P, Jiang X. Processing of autophagic protein LC3 by the 20 S proteasome. Autophagy. 2010:6:126-37.

91. Goode A, Long JE, Shaw B, Ralston SH, Visconti MR, Gianfrancesco F, Esposito T, Gennari L, Merlotti D, Rendina D, Rea SL, Sultana M, Searle MS, Layfield R. Paget disease of bone-associated UBA domain mutations of SQSTM1 exert distinct effects on protein structure and function. Biochim Biophys Acta. 1842;2014:992-1000.

92. Wei H, Wang C, Croce CM, Guan JL. p62/SQSTM1 synergizes with autophagy for tumor growth in vivo. Genes Dev. 2014;28:1204-16.

Submit your next manuscript to BioMed Central and we will help you at every step:

- We accept pre-submission inquiries

- Our selector tool helps you to find the most relevant journal

- We provide round the clock customer support

- Convenient online submission

- Thorough peer review

- Inclusion in PubMed and all major indexing services

- Maximum visibility for your research

Submit your manuscript at www.biomedcentral.com/submit 